\title{
Psycho-onco-sexologie : un ménage à trois
}

\section{Psycho-Onco-Sexology: a "ménage à trois"}

\author{
E. Marx \\ (C) Lavoisier SAS 2016
}

En septembre 2005 paraissait dans la Revue francophone de psycho-oncologie un dossier "Sexualité et cancer » [1]. En décembre 2007, à l'occasion du XXIV Congrès de la Société Française de Psycho-Oncologie dédié précisément à ce thème, notre revue lui consacrait un second dossier [2]. Entre-temps, en juillet 2006, apparaissait le terme « oncosexology " dans le titre d'une conférence organisée à Rotterdam par l'International Society for Sexuality and Cancer (ISSC) [3]. L'onco-sexologie en tant que nouvelle discipline était née, intégrant des approches médicales, psychologiques et sexologiques.

Les résultats de l'enquête « La vie deux ans après le diagnostic de cancer » [4] ont souligné la prévalence importante des troubles sexuels, $65 \%$ des hommes et des femmes interrogés déclarant des conséquences négatives du cancer sur la vie sexuelle.

Il devenait évident de devoir intégrer à la prise en charge du cancer, de l'annonce à l'après-cancer, l'information et la prise en compte des difficultés sexuelles liées à la maladie et aux effets secondaires des traitements, les recommandations des Plans cancer successifs allant dans ce sens $[5,6]$.

L'onco-sexologie est une offre de soins qui répond à une réalité. Elle concerne tous les âges et suggère une approche anticipée, évaluée et réévaluée, globale et pluridisciplinaire.

Tenir compte des troubles sexuels revient à la fois à traiter la fonction sexuelle et à aborder la sexualité. Les différentes formes de prise en charge de ces troubles sont liées à la

\footnotetext{
E. Marx $(\bowtie)$

Psychologue, sexologue

Centre Paul-Strauss, chef de l'unité de psycho-oncologie, consultation d'onco-sexologie, 3, rue de la Porte-de-l'Hôpital, BP 30042, F-67065 Strasbourg cedex, France

e-mail : emarx@strasbourg.unicancer.fr
}

Strasbourg Oncologie Libérale, centre de radiothérapie de la Robertsau, 184 , route de la Wantzenau, F-67000 Strasbourg, France

Responsable du groupe de travail « Onco-Sexologie » de l'Association Interdisciplinaire post Universitaire de Sexologie (AIUS) conception de la sexualité selon les modalités d'approche des différents professionnels impliqués en oncologie. Bien qu'elles diffèrent selon le champ d'intervention : oncologique, psychologique, sexologique, elles sont complémentaires et ne peuvent se passer l'une de l'autre. Cette collaboration permet entre autres d'aboutir à la réalisation de protocoles destinés à prévenir ou du moins à réduire les problèmes psycho-onco-sexologiques qui peuvent interférer avec l'adhésion aux traitements [7].

Où en sommes-nous aujourd'hui ? Qu'en est-il de cette alliance pluridisciplinaire entre oncologues, psychologues, psychiatres, psycho-oncologues et sexologues ou oncosexologues ? Comment les rôles et les places de chacun sont-ils partagés ? Dans quelles situations et pour quelles questions en particulier? Différents experts répondent à ces questions apportant leur éclairage et leur expérience à la saison 3 de ce dossier « Sexualité et cancer ».

La sexualité demeure encore un aspect peu abordé par les soignants. Il apparait indispensable de renforcer ou d'organiser la formation des équipes soignantes [8]. À l'instar de la douleur, il y a une vingtaine d'années, la prise en compte des troubles sexuels ne doit plus s'accompagner du silence et de l'évitement des soignants.

Pouvoir proposer une formation adaptée, destinée aux professionnels exerçant en oncologie afin de leur donner des outils de réponse et d'intervention dans le champ de la sexualité, est une question traitée par Éric Huyghe et ses collègues qui dressent un état des lieux de l'offre de formation en onco-sexologie. À l'échelon national, des formations existent à tous les niveaux. L'accent est mis sur la nécessité d'apporter des connaissances solides aux professionnels de terrain en oncologie, un alliage de savoir-faire et de savoirêtre [9].

La revue de la littérature proposée par Émilie Moreau, Pierre Moulin et Alain Giami porte sur l'évolution des liens entre cancer et sexualité. Celle-ci est traversée par un mouvement d'inversion des rapports de causalité entre cancer et sexualité. Dans le contexte actuel du concept de santé sexuelle, les auteurs établissent un parallèle avec l'apparition de l'onco-sexologie [10]. Dans un ouvrage récent, leurs 
recherches, à propos des rôles et des fonctions dévolus aux groupes professionnels impliqués dans la prise en charge des patients, ont bien montré la dichotomie existant entre la fonction sexuelle et la sexualité, au centre de la problématique sexualité et cancer [11].

Pierre Moulin et ses collaborateurs rendent compte toujours à propos des liens réciproques entre le cancer et la sexualité, des théories particulières élaborées par les infirmières, celles-ci étant parmi les professionnels de santé les moins formées dans le domaine de la sexualité tout en y étant les plus confrontées [12].

Christine Kerr et ses collègues à Montpellier soulignent la nécessité d'une approche pluridisciplinaire destinée à formaliser la prise en charge des troubles de la sexualité chez des femmes atteintes de cancers pelviens au sein d'un programme « Éducation Thérapeutique du Patient » (ETP) [13].

À l'occasion du diagnostic et des traitements du cancer, des traumatismes de violence sexuelle subis dans l'enfance peuvent ressurgir. Eliane Marx montre l'importance de la prise en charge psycho-onco-sexologique qui ne peut se passer de communication et d'échanges avec les équipes soignantes [14].

Le cancer de la prostate représente encore un tabou pour les hommes qui en sont atteints au point de museler la parole du sujet. Anne-Sophie Van Doren, Marc-Olivier Bitker et Benoît Verdon présentent les premiers résultats d'une recherche en psychologie clinique, ciblée sur les réaménagements narcissiques et identificatoires [15].

Étienne Garaud-Castillo, quant à lui, explique en quoi l'inscription à un groupe de parole constitue un grand pas pour les hommes, leur permettant de partager avec des semblables la blessure intime dont ils sont atteints [16].

En l'espace de dix ans, de nombreux professionnels de santé ont été sensibilisés à la dimension onco-sexologique. Certains sont plus réactifs que proactifs [17]. Des défis restent à relever et l'un des paradoxes réside encore dans la forte attente des patients face à une trop faible prise en charge onco-sexologique [18].

La sexualité est une composante inhérente de la qualité de vie. Il demeure essentiel que sa prise en compte dans le parcours de soins reste personnalisée, adaptée à la demande de chacun. Cela répond à des principes éthiques sans induire une médicalisation excessive de l'humain.

\section{Références}

1. Dossier «Sexualité et cancer»(2005) Rev Francoph PsychoOncologie 4:139-206

2. Dossier « Sexualité et cancer» (2007) Psycho-Oncologie 1:211-310

3. The First Rotterdam Symposium on Cancer and Sexuality (ISSC), Rotterdam (2006) Oncosexology: a new discipline? http://www. issc.nu/index.php?page $=$ oncosexology-a-new-discipline

4. Préau M, Bouhnik AD, Mancini J, et al (2008) Les difficultés sexuelles à la suite de la maladie et des traitements. In: Le Corroller-Soriano AG, Malavolti L, Mermilliod C (eds) La vie deux ans après le diagnostic de cancer. La Documentation française, Collection Études et statistiques, Paris, pp 299-310

5. http://www.e-cancer.fr/Plan-cancer/Les-Plans-cancer-de-2003-a2013

6. http://www.e-cancer.fr/Expertises-et publications/Catalogue-despublications/Plan cancer-2014-2019

7. Shover LR, van der Kaaij M, van Dorst E, et al (2014) Sexual dysfunction and infertility as late effects of cancer treatment. EJC Suppl 12:41-53

8. Bouhnik AD, Mancini J (2014) Sexualité, vie affective et conjugale. In: La vie deux ans après un diagnostic de cancer - De l'annonce à l'après-cancer. Collection Études et enquêtes, INCa, Paris, pp 418-31

9. Huyghe É, Almont T, Burte C, et al (2016) Formations en oncosexologie : existant et perspectives. Psycho-Oncologie 10:85-90

10. Moreau É, Moulin P, Giami A (2016) L'évolution des liens entre cancer et sexualité : revue critique de la littérature. PsychoOncologie 10:91-7

11. Giami A, Moreau É, Moulin P (2015) Infirmières et sexualité : entre soins et relations. Éditions de l'EHESP, Rennes

12. Moulin P, Moreau É, Giami A (2016) Sexualité et cancer : un oxymore? Psycho-Oncologie 10:98-104

13. Kerr C, Stoebner-Delabarre A, Huteau ME, Bonnabel L (2016) Cancers pelviens féminins, troubles de la sexualité, éducation thérapeutique. Psycho-Oncologie 10:105-8

14. Marx E. (2016) Lorsque le cancer et ses traitements réactivent les traumatismes sexuels subis dans l'enfance. Psycho-Oncologie 10:109-16

15. Van Doren A-S, Bitker M-O, Verdon B (2016) Détresse interdite dans le cancer de la prostate. Psycho-Oncologie 10:117-21

16. Garand-Castillo É (2016) Vivre un cancer de la prostate en 2015 : expérience d'un groupe de parole au masculin. Psycho-Oncologie 10:122-25

17. Bondil P, Habold D (2012) Cancer et sexualité : les médecins ne doivent plus faire l'impasse. La lettre du cancérologue 21:165-70

18. Bondil P, Habold D (2015) Développement de l'oncosexualité et de l'oncofertilité en France : pourquoi maintenant ? Aspects culturels et psychologiques. Psycho-Oncologie 9:230-9 